\title{
Reduced Sensitivity to Charge Noise in Semiconductor Spin Qubits via Symmetric Operation
}

\author{
M. D. Reed, B. M. Maune, R. W. Andrews, M. G. Borselli, K. Eng, M. P. Jura, A. A. Kiselev, \\ T. D. Ladd, S. T. Merkel, I. Milosavljevic, E. J. Pritchett, M. T. Rakher, R. S. Ross, \\ A. E. Schmitz, A. Smith, J. A. Wright, M. F. Gyure, and A. T. Hunter \\ HRL Laboratories, LLC, 3011 Malibu Canyon Road, Malibu, California 90265, USA
}

(Received 5 August 2015; published 16 March 2016)

\begin{abstract}
We demonstrate improved operation of exchange-coupled semiconductor quantum dots by substantially reducing the sensitivity of exchange operations to charge noise. The method involves biasing a double dot symmetrically between the charge-state anticrossings, where the derivative of the exchange energy with respect to gate voltages is minimized. Exchange remains highly tunable by adjusting the tunnel coupling. We find that this method reduces the dephasing effect of charge noise by more than a factor of 5 in comparison to operation near a charge-state anticrossing, increasing the number of observable exchange oscillations in our qubit by a similar factor. Performance also improves with exchange rate, favoring fast quantum operations.
\end{abstract}

DOI: 10.1103/PhysRevLett.116.110402

Gated semiconductor quantum dots are a leading candidate for quantum information processing due to their high speed, density, and compatibility with mature fabrication technologies $[1,2]$. Quantum dots are formed by spatially confining individual electrons using a combination of material interfaces and nanoscale metallic gates. Although several quantized degrees of freedom are available [3-5], the electron spin is often employed as a qubit due to its long coherence time [6,7]. Spin-spin coupling may be controlled via the kinetic exchange interaction, which has the benefit of short range and electrical controllability. Numerous qubit proposals use exchange, including as a two-qubit gate between ESR-addressed spins [8], a single axis of control in a two dot system also employing gradient magnetic fields [9] or spin-orbit couplings [10], or as a means of full qubit control on a restricted subspace of at least three coupled spins [11-13]. However, since exchange relies on electron motion, it is susceptible to electric field fluctuations, or charge noise. Limiting the consequence of this noise is critical to attaining performance of exchange-based qubits adequate for quantum information processing.

Charge noise in semiconductor quantum dots may originate from a variety of sources including electric defects at interfaces and in dielectrics [14]. These defects typically result in electric fields that exhibit an approximate $1 / f$ noise spectral density. Conventional routes for reducing charge noise include improving materials and interfaces [15] and dynamical decoupling [16-19]. In this Letter,

Published by the American Physical Society under the terms of the Creative Commons Attribution 3.0 License. Further distribution of this work must maintain attribution to the author(s) and the published article's title, journal citation, and DOI. rather than addressing the microscopic origins or detailed spectrum of charge noise, we introduce a "symmetric" mode of operation where the exchange interaction is less susceptible to that noise. This is done by biasing the device to a regime where the strength of the exchange interaction is first-order insensitive to dot chemical potential fluctuations but is still controllable by modulating the interdot tunnel barrier. This dramatically reduces the effects of charge noise.

The principle of symmetric operation can be understood by treating charge noise as equivalent to voltage fluctuations on confinement gates. This approximation is valid when materials or interfaces proximal to gates are the dominant source of noise [14]. In this context, noise sensitivity may be reduced by biasing the device to a "sweet spot" where small changes in gate voltages only weakly alter the strength of the exchange interaction. Previously explored methods include using a triple quantum dot with balanced exchange interactions [20,21] (see the Supplemental Material [22] for a comparison), operating far from the $(1,1)$ charge regime where excited states flatten the exchange profile $[21,23,24]$, using double dots populated with more than two electrons [25], or tailoring exchange derivatives via magnetic field gradients [26]. The strategy we pursue in this Letter has the advantage of employing only pairwise exchange without requiring high or inhomogeneous magnetic fields and maintains tunability of the exchange coupling rate from being negligibly small to many $\mathrm{GHz}$.

Symmetric operation is diagrammed in Fig. 1. The difference in chemical potential between two dots is denoted $\Delta$ and is predominantly controlled by two gates labeled $P 1$ and $P 2$ in Fig. 1(a). For an ideal double quantum dot, $\Delta=\alpha\left(V_{P 1}-V_{P 2}\right)$ where $\alpha$ is the "lever arm" that converts voltage to chemical potential. A third 

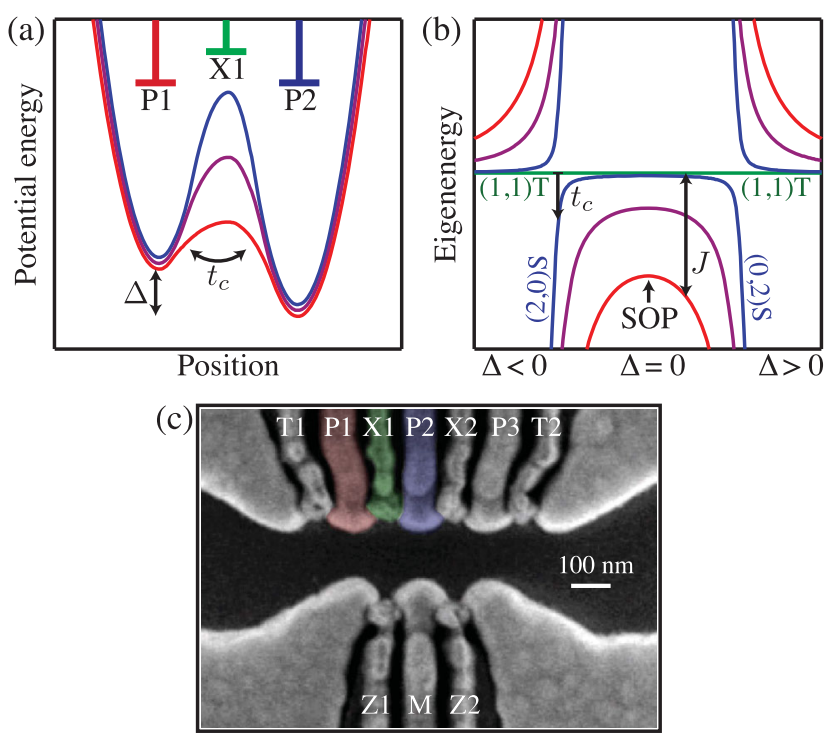

FIG. 1. (a) Schematic of a double-dot potential energy as a function of position, in which the left dot has a chemical potential higher than the right dot by the detuning $\Delta$. The chemical potentials are predominantly controlled by gates $P 1$ and $P 2$ (schematized above each) and the barrier by $X 1$. When $X 1$ is forward biased, the tunnel coupling $t_{c}$ is increased, with the blue curve indicating low $t_{c}$ and the red curve high $t_{c}$. (b) Schematic of eigenenergies of the double dot as a function of $\Delta$, according to a simple Hubbard model. Here, the singlet states are again colored blue to red as $t_{c}$ is increased, while the triplet states are colored green. Exchange $J$ is the difference between singlet and triplet energies; $\partial J / \partial \Delta$ vanishes at the symmetric operating point $\Delta=0$. Note that our definition of $\Delta$ corresponds to the chemical potential difference of singly occupied dots and not distance from the anticrossing (typically notated $\varepsilon$ as in Refs. [2,13,27]). (c) Representative electron micrograph of a triple quantum dot, with gate traces labeled. We false color gates $P 1, X 1$, and $P 2$, respectively, red, green, and blue.

gate, labeled $X 1$, controls the potential barrier that sets the rate at which an electron can tunnel, $t_{c} / h$. Figure 1(b) shows eigenenergies for a double dot as calculated with a Hubbard model. Crucially, although the detuning $\Delta$ is often used to control $J$, the tunnel coupling $t_{c}$ can also modify the energy difference between the singlet and triplet energy eigenstates, $J\left(\Delta, t_{c}\right)$. In particular, $J\left(\Delta=0, t_{c}\right)$ is a "sweet spot" where the effects of charge noise on exchange are reduced because $\partial J / \partial \Delta=0[28,29]$, as evident from Fig. 1(b). We refer to $J\left(\Delta=0, t_{c}\right)$ as a symmetric operating point (SOP) because the double quantum dot is biased to the center of the $(1,1)$ charge cell and equidistant from the $(2,0)$ and $(0,2)$ anticrossings.

Although any system of exchange-coupled quantum dot pairs could potentially benefit from symmetric operation, we use Si-based triple-quantum-dot devices for our demonstration. A SEM image of a device is shown in Fig. 1(c). Metallic plunger gates $P 1-P 3$ are deposited on an undoped $\mathrm{Si} / \mathrm{SiGe}$ heterostructure. When the plungers are forward biased, individual electrons are drawn from a bath and accumulate beneath the plungers $[13,15]$. The $X$ and $T$ gates are deposited on an insulating layer that overlaps the plungers and control tunnel barriers between the dots and to the electron bath. Some devices in our study differ from Ref. [13] by the addition of a metal screening gate which prevents charge accumulation under gate leads [30]. A proximal dot charge sensor formed by the $M$ and $Z$ gates enables single-shot readout of the qubit state [13]. $P$ and $X$ gate control lines are capable of nanosecond pulse rise times and amplitudes of $140 \mathrm{mV}$. The devices are operated in a dilution refrigerator, giving $T_{e} \sim 100 \mathrm{mK}$.

The third dots in our devices enable initialization and measurement [see Fig. 2(a) of Ref. [13] ]. In the experiments described below, we prepare the qubit state by biasing near the $(1,0,1)-(1,0,2)$ charge transition where a two-electron singlet state is preferentially loaded into dot 3 . One of the electrons is then transferred into dot 2 by ramping $P 2$ and $P 3$. We define this singlet state between dots 2 and 3 as the north pole of a qubit Bloch sphere [11]. Exchange between dots 1 and 2 occurs at a frequency $J\left(\Delta, t_{c}\right) / h=J(\mathbf{V}) / h$, where $\mathbf{V}$ denotes the gate voltages. This interaction rotates the qubit state about an axis which is tipped $120^{\circ}$ from the north pole [11-13]. After some evolution, we measure the qubit state using Pauli blockade by biasing near the $(1,0,2)-(1,1,1)$ charge transition. Sweeping the evolution time yields Rabi oscillations which have a maximum contrast of $75 \%$ due to the tilted rotation axis.

We demonstrate singlet-triplet Rabi oscillations in Fig. 2(a) by sweeping the exchange duration and $\Delta$ while holding $t_{c}$ constant. The Rabi frequency is given by $J\left(\Delta, t_{c}\right) / h$ and is large even with $\Delta=0$ because $V_{X 1}$ is forward biased during evolution, increasing $t_{c}$. $J$ increases with $|\Delta|$, producing a chevron pattern. The number of resolvable oscillations is greatest at the $\operatorname{SOP}(\Delta=0)$, giving preliminary indication that using a SOP can enhance the quality of the exchange interaction. This improvement can be interpreted in the context of gate-referred charge noise. As discussed in the Supplemental Material [22], for large detuning $|d J / d \Delta| \approx J^{2} / t_{c}^{2}$. Thus, as $J$ is increased by detuning, it becomes quadratically more susceptible to charge noise. When $\Delta=0$, however, the dominant derivative is now $d J / d V_{X 1}=\left(\partial J / \partial t_{c}\right)\left(d t_{c} / d V_{X 1}\right)$, proportional only to $J$. Increasing $J$ with $t_{c}$, then, only linearly increases susceptibility to charge noise. (This scaling is valid when $J$ is exponentially dependent on $V_{X 1}$; later, we show that it can be subexponential and, thus, even more favorable.)

The shape of the Rabi oscillations shown in Fig. 2(b) can be modeled with a two-channel decay process. One process is due to the hyperfine interaction between the electron spin and that device's natural abundance of ${ }^{29} \mathrm{Si}$ nuclei and is described by Eq. (12) of Ref. [31]. The other process is due to charge noise, which, for the $1 / f$ spectrum of noise seen in these devices [13], imposes a Gaussian envelope. For this illustrative example, the relatively low value of $J$ and the 

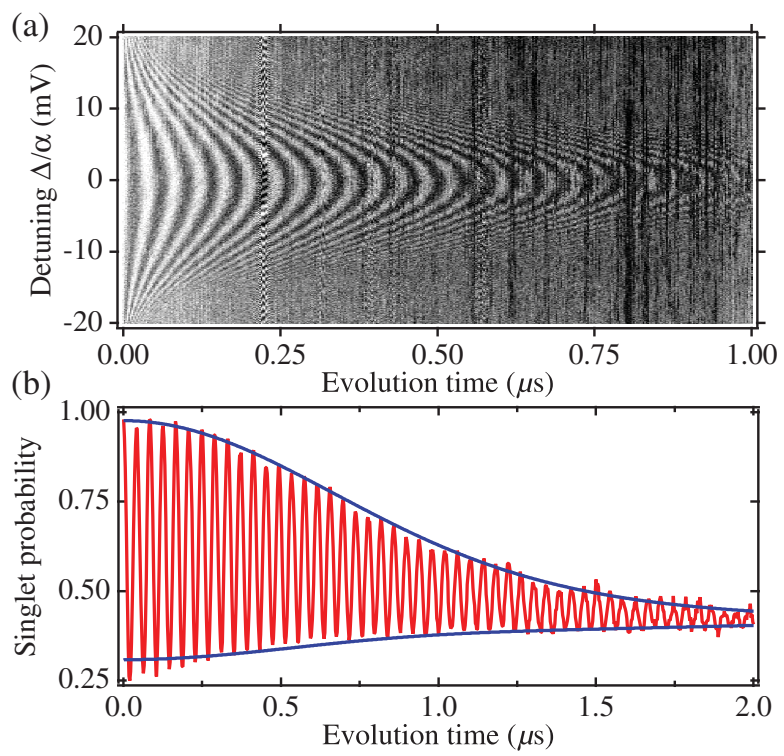

FIG. 2. Rabi oscillations at a SOP. (a) We observe exchange oscillations by biasing to a detuning ( $y$ axis) for a given time ( $x$ axis). The middle of the plot, where $\Delta=0$ and $J$ is minimized, corresponds to the SOP. The gray scale measures singlet probability and ranges from $100 \%$ (white) to $25 \%$ (black). (b) Evolving at $\Delta=0$ reveals a time-domain Rabi oscillation showing a double Gaussian decay with a $1 / e$ time of $1.0 \mu$ s due to hyperfine interactions and $1.5 \mu$ s due to charge noise.

natural isotopic abundance of this sample renders the charge decoherence time comparable to the magnetic dephasing time. In the discussion that follows, however, because we focus on higher values of $J$ in isotopically purified silicon samples, charge noise will dominate the decay envelope.

For gate-referred $1 / f$ charge noise, this envelope can be expressed as $\exp \left(-\sigma_{V}^{2} \sum_{j}\left|d J / d V_{j}\right|^{2} t^{2} / \hbar^{2}\right)$. Here, $\sigma_{V}^{2}$ is the variance of the noise (e.g., the noise spectral density integrated over relevant time scales) and $j$ indexes all gates; see Ref. [23] and the Supplemental Material [22]. Increasing the Rabi decay time for fixed $J$ can then be accomplished by reducing $\sum_{j}\left|d J / d V_{j}\right|^{2}$ [28]. We define the insensitivity $\mathcal{I}$ as

$$
\mathcal{I}=J / \sqrt{\sum_{j}\left|d J / d V_{j}\right|^{2}}
$$

which has units of voltage. With this metric, the expected envelope of Rabi oscillations is $\exp \left[-(J t / \hbar)^{2}\left(\sigma_{V} / \mathcal{I}\right)^{2}\right]$, so that the number of oscillations that occur before the amplitude decays by $1 / e$ is $\mathcal{I} /\left(2 \pi \sigma_{V}\right)$. As long as $\sigma_{V}$ is not too strong a function of control parameters (e.g., noise not varying from one gate to the next), we can optimize device performance by maximizing $\mathcal{I}$ with respect to $\mathbf{V}$. In particular, only the charge noise variance and not the detailed structure of its spectral density is relevant to this calculation, enabling predictions of bias-dependent charge noise performance based on device electrostatics.

In order to demonstrate the advantage of symmetric operation, we must independently control $\Delta$ and $t_{c}$. The plunger and exchange gates affect both parameters due to capacitive cross talk. Using a routine described in the Supplemental Material [22], we orthogonalize these control axes. Modulation of $t_{c}$ is accomplished by changing $V_{X 1}$ along with small compensating voltages on plunger gates, while $\Delta$ is modified by biasing $P 1$ and $P 2$ with approximately equal and opposite voltages. We show the effect of these parameters on $J$ in Fig. 3, where we evolve for a fixed time at a point that is swept in both $t_{c}$ (parametrized by $\left.V_{X 1}\right)$ and $\Delta$. This "fingerprint" plot shows fringes due to varying $J$, the curvature of which indicates where $d J / d \Delta=0$. This locus of points, which on this plot is approximately parallel to the $x$ axis due to our orthogonalization scheme, is known as the symmetric axis and indicates the location of the SOP for a given $J$.

Symmetric operation maximizes $\mathcal{I}$. To demonstrate this, we choose various combinations of $\Delta$ and $t_{c}$ where $J / h=160 \mathrm{MHz}$, shown in the inset of Fig. 4(a). At each evolution point, we explicitly measure $\mathcal{I}$ by determining how the Rabi oscillation frequency changes due to small perturbations in each of the seven relevant gate voltages. We plot the resulting insensitivity in Fig. 4(a) and find that it is maximized at $\Delta=0$ with a value of $\sim 30 \mathrm{mV}$ and rapidly decreases to less than $5 \mathrm{mV}$ for large $\Delta$.

To test the validity of $\mathcal{I}$ as a metric for the effects of charge noise, we measure the number of Rabi oscillations $N_{\text {Rabi }} \equiv J \tau / h$ that occur in a $1 / e$ decay time $\tau$. If the gatereferred charge-noise variance $\sigma_{V}^{2}$ were equal and uncorrelated on all gates, then we would expect $\mathcal{I} \propto N_{\text {Rabi }}$.

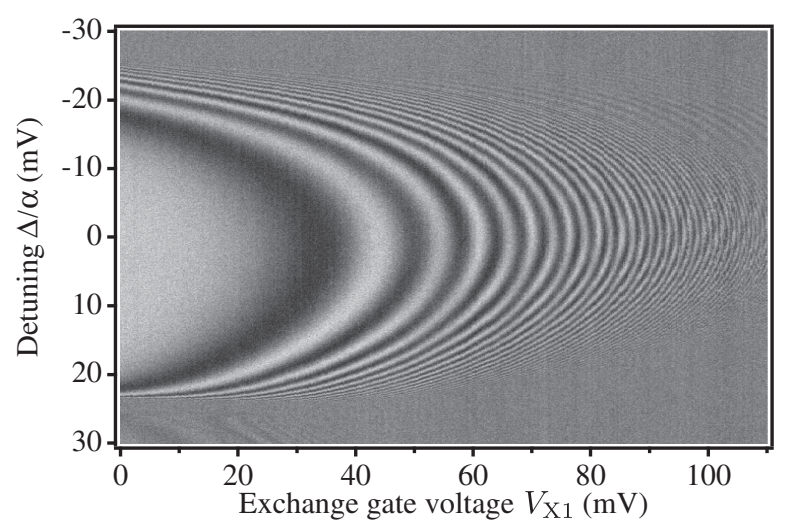

FIG. 3. Fingerprint plot demonstrating the dependence of exchange on $\Delta$ and $V_{X 1}$. We plot the average singlet probability after evolving for $500 \mathrm{~ns}$ at a potential specified by the axes. The $z$ scale is the same as in Fig. 2. A faint set of additional fringes is present in this data [prominent near $\left(\Delta, V_{X 1}\right)=(-20,100)$ ], likely due to excited state population (Supplemental Material [22]). The device used here and in all subsequent figures differs from the device used in Fig. 2 by the addition of a screening gate [30] and the use of enriched ${ }^{28} \mathrm{Si}\left(800 \mathrm{ppm}^{29} \mathrm{Si}\right)[13]$. 


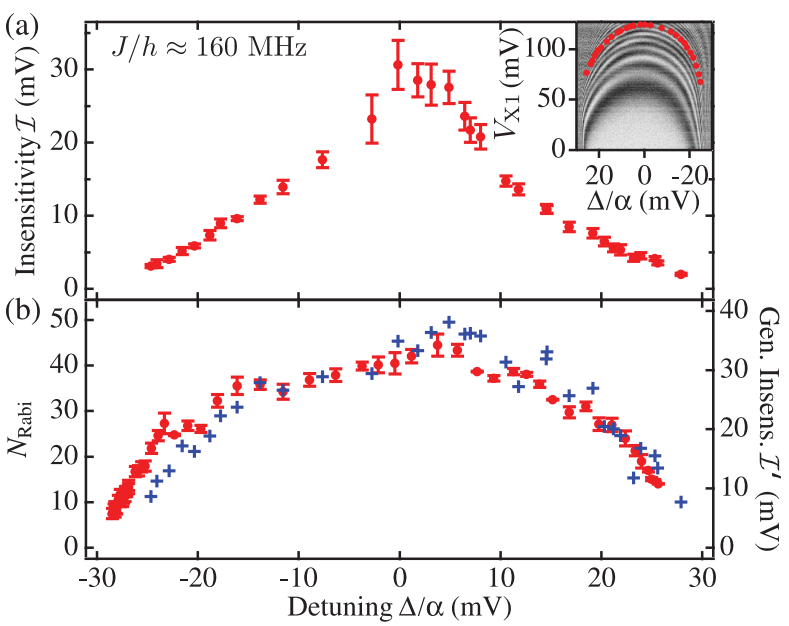

FIG. 4. Insensitivity and number of fringes along a constant- $J$ curve. (a) We measure $\mathcal{I}$ along a contour where $J / h \approx 160 \mathrm{MHz}$ for various $\Delta$ and $V_{X 1}$ (inset). $\mathcal{I}$ is peaked near $\Delta=0$ at a value of $\sim 30 \mathrm{mV}$, rapidly falling to below $5 \mathrm{mV}$ as $|\Delta|$ is increased. (b) We verify that $\mathcal{I}$ is a valid proxy for device performance by measuring the number of fringes present in a $1 / e$ decay time with a series of time-Rabi experiments where the evolution point is swept along the same contour. Because of the presence of two evolution frequencies in this device (Supplemental Material [22]), we apply a high-pass filter to the time-domain data before extracting the decay coefficient. We plot the product of that coefficient and $J / h$ as closed red circles. We also plot a generalized definition of $\mathcal{I}$ (blue crosses) which better models the data (Supplemental Material [22]).

To determine $N_{\text {Rabi }}$, we measure $\tau$ along the voltage arc where $J\left(\Delta, t_{c}\right) / h=160 \mathrm{MHz}$. The resulting $N_{\text {Rabi }}$ is plotted in Fig. 4(b) (red circles). Though it qualitatively follows $\mathcal{I}$ and is maximum near $\Delta=0$, the quantities are not strictly proportional, indicating that our assumptions are not fully supported. In particular, as discussed in the Supplemental Material [22], by including some knowledge of the relative geometries of the $P$ and $X$ gates in this device, we can more accurately model $N_{\text {Rabi }}$ with a generalized definition of $\mathcal{I}$ [blue crosses in Fig. 4(b)].

Charge noise is not the only source of degradation for exchange-based control: finite $T_{1}$ and hyperfine dephasing will also play a role. Their effects are generally diminished by making the evolution time as short as possible, requiring $J$ to be maximized. When $J$ is controlled solely by $\Delta$, this poses a major problem as $\mathcal{I}$ will drop precipitously; one must trade-off between infidelity sources. Fortunately, for symmetric operation, there is no such penalty; indeed, performance actually improves. We see this by first noting that $J$ will depend exponentially on the height of the tunnel barrier when $t_{c}$ is small. As we approach the large- $J$ limit, however, the SOP double dot merges into a larger twoelectron single dot where the former barrier is shallow. In this regime, $J$ will saturate to near that broader potential's orbital excited energy and not depend on $t_{c}$ (Supplemental

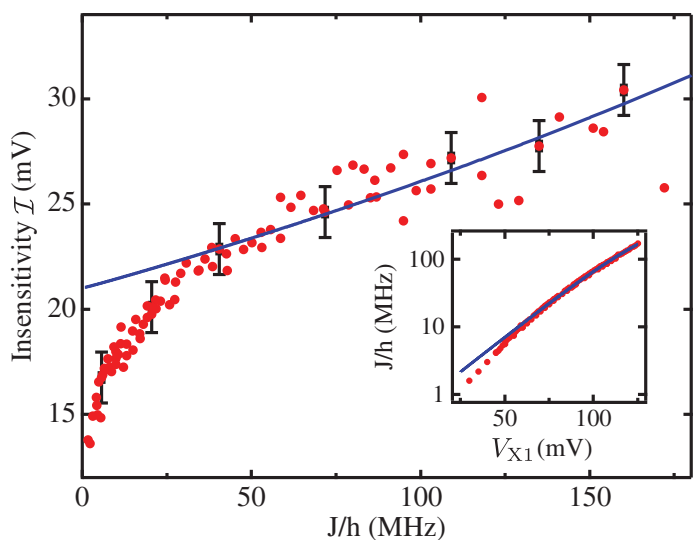

FIG. 5. Asymptotic behavior along the symmetric axis. (inset) We measure $J$ as a function of $V_{X 1}$ along the symmetric operating vector, $J\left(\Delta=0, t_{c}\right)$, and find that it is subexponential at large $J$. (main figure) As a consequence of this asymptotic behavior, $\mathcal{I}$ monotonically increases with $J$, roughly doubling over the relevant voltage range. We plot several representative error bars in black and suppress the rest for clarity. We compare the insensitivity of several similar devices in the Supplemental Material [22].

Material [22]). This is reflected in the inset of Fig. 5, where $J(\mathbf{V})$ behaves subexponentially with increasing $V_{X 1}$. Since the main contributor to $\mathcal{I}$ when $\Delta=0$ is this exchange gate, we see, in Fig. 5, that $\mathcal{I}$ monotonically increases with $J$, doubling over the selected range. We may fit this behavior at high $J$ using a one-dimensional WKB approximation appropriate for shallow barrier tunneling [32] which correctly captures the subexponential behavior of $J$ and increased $\mathcal{I}$ but breaks down at low $t_{c}$. In some cases, detailed 3D single-electron Poisson-Schrödinger simulations including disorder captures the full behavior.

We have shown that symmetric operation improves the noise characteristics of semiconductor qubits employing the exchange interaction. Compared to detuning, it is substantially less sensitive to noise, particularly for large $J$ where fast gates are possible. Though we demonstrated symmetric operation with an exchange-only Si triple dot, the principle should work equally well in any device where $\Delta$ and $t_{c}$ can be separately controlled. Indeed, we recently became aware of demonstrations in GaAs double dots $[33,34]$. Future work will be to characterize the benefits of symmetric operation on control fidelity using techniques such as randomized benchmarking.

We thank C. Jones, J. Kerckhoff, S. Meenehan, and D. Underwood for discussions. This research was developed with funding from the Defense Advanced Research Projects Agency (DARPA). The views, opinions, and/or findings contained in this material are those of the authors and should not be interpreted as representing the official views or policies of the Department of Defense or the U.S. Government. 
*athunter@hrl.com

[1] D. Loss and D. P. DiVincenzo, Quantum computation with quantum dots, Phys. Rev. A 57, 120 (1998).

[2] J. R. Petta, A. C. Johnson, J. M. Taylor, E. A. Laird, A. Yacoby, M. D. Lukin, C. M. Marcus, M. P. Hanson, and A.C. Gossard, Coherent manipulation of coupled electron spins in semiconductor quantum dots, Science 309, 2180 (2005).

[3] D. Kim, Z. Shi, C. B. Simmons, D. R. Ward, J. R. Prance, T. S. Koh, J. K. Gamble, D. E. Savage, M. G. Lagally, M. Friesen, S. N. Coppersmith, and M. A. Eriksson, Quantum control and process tomography of a semiconductor quantum dot hybrid qubit, Nature (London) 511, 70 (2014).

[4] K. D. Petersson, C. G. Smith, D. Anderson, P. Atkinson, G. A. C. Jones, and D. A. Ritchie, Charge and spin state readout of a double quantum dot coupled to a resonator, Nano Lett. 10, 2789 (2010).

[5] D. Culcer, A. L. Saraiva, B. Koiller, X. Hu, and S. Das Sarma, Valley-Based Noise-Resistant Quantum Computation Using Si Quantum Dots, Phys. Rev. Lett. 108, 126804 (2012).

[6] K. Wang, C. Payette, Y. Dovzhenko, P. W. Deelman, and J. R. Petta, Charge Relaxation in a Single-Electron $\mathrm{Si} / \mathrm{SiGe}$ Double Quantum Dot, Phys. Rev. Lett. 111, 046801 (2013).

[7] M. Veldhorst, J. C. C. Hwang, C. H. Yang, A. W. Leenstra, B. d. Ronde, J. P. Dehollain, J. T. Muhonen, F. E. Hudson, K. M. Itoh, A. Morello, and A. S. Dzurak, An addressable quantum dot qubit with fault-tolerant control-fidelity, Nat. Nanotechnol. 9, 981 (2014).

[8] M. Veldhorst, C. H. Yang, J. C. C. Hwang, W. Huang, J. P. Dehollain, J. T. Muhonen, S. Simmons, A. Laucht, F. E. Hudson, K. M. Itoh, A. Morello, and A. S. Dzurak, A twoqubit logic gate in silicon, Nature (London) 526, 410 (2015).

[9] R. Brunner, Y.-S. Shin, T. Obata, M. Pioro-Ladrière, T. Kubo, K. Yoshida, T. Taniyama, Y. Tokura, and S. Tarucha, Two-Qubit Gate of Combined Single-Spin Rotation and Interdot Spin Exchange in a Double Quantum Dot, Phys. Rev. Lett. 107, 146801 (2011).

[10] K. C. Nowack, F. H. L. Koppens, Y. V. Nazarov, and L. M. K. Vandersypen, Coherent control of a single electron spin with electric fields, Science 318, 1430 (2007).

[11] D. P. DiVincenzo, D. Bacon, J. Kempe, G. Burkard, and K. B. Whaley, Universal quantum computation with the exchange interaction, Nature (London) 408, 339 (2000).

[12] J. Medford, J. Beil, J. M. Taylor, S. D. Bartlett, A. C. Doherty, E. I. Rashba, D. P. DiVincenzo, H. Lu, A.C. Gossard, and C. M. Marcus, Self-consistent measurement and state tomography of an exchange-only spin qubit, Nat. Nanotechnol. 8, 654 (2013).

[13] K. Eng, T. D. Ladd, A. Smith, M. G. Borselli, A. A. Kiselev, B. H. Fong, K. S. Holabird, T. M. Hazard, B. Huang, P. W. Deelman, I. Milosavljevic, A. E. Schmitz, R. S. Ross, M.F. Gyure, and A. T. Hunter, Isotopically enhanced triple-quantum-dot qubit, Sci. Adv. 1, e1500214 (2015).

[14] E. Paladino, Y. M. Galperin, G. Falci, and B. L. Altshuler, $1 / f$ noise: Implications for solid-state quantum information, Rev. Mod. Phys. 86, 361 (2014).
[15] M. G. Borselli, K. Eng, R. S. Ross, T. M. Hazard, K. S. Holabird, B. Huang, A. A. Kiselev, P. W. Deelman, L. D. Warren, I. Milosavljevic, A. E. Schmitz, M. Sokolich, M. F. Gyure, and A. T. Hunter, Undoped accumulationmode Si/SiGe quantum dots, Nanotechnology 26, 375202 (2015).

[16] X. Wang, L.S. Bishop, E. Barnes, J. P. Kestner, and S. Das Sarma, Robust quantum gates for single-triplet spin qubits using composite pulses, Phys. Rev. A 89, 022310 (2014).

[17] P. Cerfontaine, T. Botzem, D. P. DiVincenzo, and H. Bluhm, High-Fidelity Single-Qubit Gates for Two-Electron Spin Qubits in GaAs, Phys. Rev. Lett. 113, 150501 (2014).

[18] C. Kabytayev, T. J. Green, K. Khodjasteh, M. J. Biercuk, L. Viola, and K. R. Brown, Robustness of composite pulses to time-dependent control noise, Phys. Rev. A 90, 012316 (2014).

[19] X. Wang, F. A. Calderon-Vargas, M. S. Rana, J. P. Kestner, E. Barnes, and S. Das Sarma, Noise-compensating pulses for electrostatically controlled silicon spin qubits, Phys. Rev. B 90, 155306 (2014).

[20] J. M. Taylor, V. Srinivasa, and J. Medford, Electrically Protected Resonant Exchange Qubits in Triple Quantum Dots, Phys. Rev. Lett. 111, 050502 (2013).

[21] J. Fei, J.-T. Hung, T. S. Koh, Y.-P. Shim, S. N. Coppersmith, $\mathrm{X}$. Hu, and M. Friesen, Characterizing gate operations near the sweet spot of an exchange-only qubit, Phys. Rev. B 91, 205434 (2015).

[22] See Supplemental Material at http://link.aps.org/ supplemental/10.1103/PhysRevLett.116.110402 for additional technical details and theoretical calculations.

[23] O. E. Dial, M. D. Shulman, S. P. Harvey, H. Bluhm, V. Umansky, and A. Yacoby, Charge Noise Spectroscopy Using Coherent Exchange Oscillations in a Singlet-Triplet Qubit, Phys. Rev. Lett. 110, 146804 (2013).

[24] T. Hiltunen, H. Bluhm, S. Mehl, and A. Harju, Charge-noise tolerant exchange gates of singlet-triplet qubits in asymmetric double quantum dots, Phys. Rev. B 91, 075301 (2015).

[25] A. P. Higginbotham, F. Kuemmeth, M. P. Hanson, A. C. Gossard, and C. M. Marcus, Coherent Operations and Screening in Multielectron Spin Qubits, Phys. Rev. Lett. 112, 026801 (2014).

[26] C. H. Wong, M. A. Eriksson, S. N. Coppersmith, and M. Friesen, High-fidelity singlet-triplet $S-T_{\text {_ }}$ qubits in inhomogeneous magnetic fields, Phys. Rev. B 92, 045403 (2015).

[27] B. M. Maune, M. G. Borselli, B. Huang, T. D. Ladd, P. W. Deelman, K. S. Holabird, A. A. Kiselev, I. AlvaradoRodriguez, R. S. Ross, A. E. Schmitz, M. Sokolich, C. A. Watson, M. F. Gyure, and A. T. Hunter, Coherent singlettriplet oscillations in a silicon-based double quantum dot, Nature (London) 481, 344 (2012).

[28] J. M. Taylor, J. R. Petta, A. C. Johnson, A. Yacoby, C. M. Marcus, and M.D. Lukin, Relaxation, dephasing, and quantum control of electron spins in double quantum dots, Phys. Rev. B 76, 035315 (2007).

[29] J. Klinovaja, D. Stepanenko, B. I. Halperin, and D. Loss, Exchange-based CNOT gates for singlet-triplet qubits with spin-orbit interaction, Phys. Rev. B 86, 085423 (2012). 
[30] D. M. Zajac, T. M. Hazard, X. Mi, K. Wang, and J. R. Petta, A reconfigurable gate architecture for $\mathrm{Si} / \mathrm{SiGe}$ quantum dots, Appl. Phys. Lett. 106, 223507 (2015).

[31] T. D. Ladd, Hyperfine-induced decay in triple quantum dots, Phys. Rev. B 86, 125408 (2012).

[32] S. K. Bhattacharya and A. R. P. Rau, Coulomb spectrum in crossed electric and magnetic fields: Eigenstates of motion in double-minimum potential wells, Phys. Rev. A 26, 2315 (1982).

[33] B. Bertrand, H. Flentje, S. Takada, M. Yamamoto, S. Tarucha, A. Ludwig, A. D. Wieck, C. Bäuerle, and T. Meunier, Quantum Manipulation of Two-Electron Spin
States in Isolated Double Quantum Dots, Phys. Rev. Lett. 115, 096801 (2015).

[34] F. Martins, F. K. Malinowski, P. D. Nissen, E. Barnes, S. Fallahi, G. C. Gardner, M. J. Manfra, C. M. Marcus, and F. Kuemmeth, this issue, Noise Suppression using Symmetric Exchange Gates in Spin Qubits, Phys. Rev. Lett. 116, 116801 (2016); E. Barnes, M. S. Rudner, F. Martins, F. K. Malinowski, C. M. Marcus, and F. Kuemmeth, Filter function formalism beyond pure dephasing and non-Markovian noise in singlet-triplet qubits, Phys. Rev. B 93, 121407(R) (2016). 